\title{
The Changing Landscape of Ageing Workforce in Hong Kong - The Importance of ICT Training in Lifelong Corporate Learning
}

\author{
doi:10.3991/ijac.v3i1.1171 \\ S. Lam ${ }^{1}$ and W. Chung ${ }^{2}$ \\ ${ }^{1}$ Oxford University, Oxford, UK \\ ${ }^{2}$ Tung Wah Eastern Hospital, Hong Kong
}

\begin{abstract}
To analyse the changing landscape of ageing workforce in Hong Kong and its needs of ICT training at both the individual worker level and the firm level. This study describes the decisions that older workers are actually making about work and retirement in order to understand the changing expectation of older employees when they reach the retirement age. A large scale of a purposive sample of over 500 aged 50 years or older employers completed an on-line survey. A selected sub-sample of 50 participated in follow-up in-depth interviews. It indicates that older workers are willing to contribute, but in a more flexible way and willing to learn ICT skills to retain active for both social and employment reasons. One important implication for many employers is that they have to redefine specific recruiting, retention and workplace strategies to address the different needs, priorities and preferences among older workers, and how to keep them productive and satisfied.
\end{abstract}

Index Terms-ICT, Ageing workforce, Hong Kong, Corporate Training

\section{INTRODUCTION}

Demographic change will continue to drastically alter the structure of the workforce in coming decades in almost all developed countries. This will lead to a diminishing supply of labour, which could have an impact on productivity and competitiveness. Societies are significantly affected by the low employment rates of older workers. When workers retire early, dependency ratios (the proportion of 15- to 64-year-olds in relation to other age groups) increase and an increasingly heavy burden develops with the costs of retirement and health care growing. Ageing also challenges the sufficiency and quality of social and health care services, including occupational health services. Work life must be lengthened for the sake of society, otherwise we cannot afford to grow older [1] [2]. A greater understanding is needed of how the labour market and the working lives of people will need to adapt to an "ageing workforce" that also has to cope with technological changes and an increasingly global economy. During the 1990s the trend was for older workers to be excluded from the labour market. Increasingly there will be a need to reverse this trend in order to cope with these demographic changes. Education and training systems are going to need to adjust to these changes in order to ensure older workers, have the necessary competence to meet these new demands, perhaps, on their longer working lives - thus the growing importance of lifelong learning.

There is a widespread belief that older people have a more negative attitude toward automation than younger people. Some studies have revealed differences between age groups in the ways of learning data systems. However, stereotyped attitudes dominate the discussion about ageing people and computers. When new computer applications are implemented, the right timing of the computer training and more time to get practice in real daily work situations are of crucial importance. Information overload is a risk especially under poor ergonomic conditions. With increasing age, elimination of the impacts of distracting factors in the environment, typically noise and poor lighting conditions, is more difficult. These hamper concentration ability and direction of attention, which in turn may lead to errors in memory functions, e.g. material to be learned is not processed in the short-term memory. The most problematic situation is among the less-educated elderly workers in routine tasks. They have had fewer possibilities for positive learning experiences and career development. Age-conscious personnel policy means that company-specific measures should be planned for all age groups.

A comprehensive solution for the ageing challenge at the level of enterprise is the promotion of work ability during ageing [2]. This concept focuses on both human resources and working conditions. The core dimensions of human resources include health, physical and mental capacities and social functioning, competencies, as well as attitudes and values. The core dimensions of work cover the contents and demands of work, physical, ergonomic and psychosocial work environment, as well as management and leadership issues. Work ability is also connected to the microenvironment outside the workplace (family, relatives, friends, etc) as well as with the macro environment (infrastructure, services and other societal dimensions) [3][4][5]. The new core concept of work ability emphasizes the balance between human resources and work. Because the employee is mainly responsible for his / her resources and the employer for the dimensions of work, good work ability can be achieved only by better cooperation between the supervisor and employee. The challenge today is that the human resources will change with age and, simultaneously, the globalization and new technology will change the work. The balance leading to 
good work ability should be achieved in a dynamic, continuous changing process during the work career.

Modern ICTs play an important role as tools contributing to work-life balance and to the reconciliation of employment/work and other life spheres and activities. Under the heading of "Active Ageing" - a debate has emerged that high-lights the positive contribution older adults are able to make to both society and economy rather than merely associating the phenomenon of population ageing with a socie-tal/economic "burden”. For instance, the OECD defines active ageing as "... the capacity of people, as they grow older, to lead productive lives in the society and the economy. This means that they can make flexible choices in the way they spend time over life - in learning, in work, in leisure and in care-giving". Up to now, this debate has largely focused on socioeconomic issues. Key features of this debate include for instance an ageing work force, pressure for increased employment rates among the older segments of the population and pressure towards later retirement. However, not much attention has yet been paid to the relationship between the need of ICT skills / training and an ageing workforce.

\section{A. Business Attitudes to Ageing}

This changing demographic profile has major implications for Hong Kong's employers :

- More older workers will be available than younger ones;

- Older workers will stay in the labour market longer;

- Education and training needs will change;

- More flexible working practices will be required;

- Opportunities for competitive advantage for those companies that become 'age-friendly' employers.

On the whole, firms in Hong Kong have yet to embrace the above changes. Our other related research found that while firms are starting to recognise demographic change as one of their biggest challenges after globalisation, many have not fully analysed their employee age structure and most still see personnel planning as a short term activity. On average, companies have a one year time horizon in their staff planning. Most are not geared up to age friendly employment policies in areas such as lifelong learning, career management, healthcare, knowledge management, and age diversity management.

\section{Methodology}

This paper addresses the latest trend in ageing workforce by understanding the need of ICT skills through an analysis of a survey. It consisted both of an interactive online survey and face-to-face interview: a current and comprehensive investigation of public attitudes in Hong Kong toward the need of ICT skills in ageing workforce. To qualify for the study, participants had to be between the ages of 50-65.

This study examined needs of ICT training in various aspects of elderly citizen. Key questions included:

- training and e-skills development for older people

- the contribution made by ICT to flexible work and retirement schemes and working conditions for older people
- The contribution of ICT - such as via e-working and e-learning - to improved professional opportunities and work-life balance.

- e-access to supportive resources and networks for atrisk groups, especially those with caring responsibilities

A primary focus of the 50-question survey was to better understand the experiences and behaviors of the aging workforce, so the survey included many questions asked only of people who were still working or seeking work, either full- or part-time. To support the survey data, additional interviews were conducted with people who fit the survey profile, as well as with company managers responsible for workforce diversity and experts in recruiting older workers. The study entailed face-to-face interviews with its nationally representative random sample. Personal interviews were conducted through household visits, based on a multi-stage sampling technique designed to provide a sample that could yield estimates for the population as a whole. A probability sample of households was drawn up as the basis for visits. Field staffs then randomly sampled individuals within households to determine whom to interview. The aim was to obtain 50 completed interviews. An excellent response rate of $80 \%$ of those contacted yielded 40 respondents. The interviews lasted 30 to 35 minutes on average, covering a wide range of topics related to their ICT ability and job situation, as well as the background of the respondent. Interviews began with general questions, such as attitudes about the Internet and technology in general, before proceeding to detailed questions about the use of ICT knowledge in their job. Interviews were also conducted in different focus groups: ICT providers, course operators, and voluntary organizations. This was necessary to extend the range of the research and to minimise the risk of relying on the inevitably one-sided perspective of any particular social actor.

\section{RESULTS}

\section{A. Motivations for Meeting Changing Opportunities}

The number one reason for taking retirement benefits cited by workers age 60-65 was "wanted to try something new and different with the latest technology" (20\%). This option was chosen much less frequently by $50-55$ year olds (10\%) and by 56-59 year-olds (10\%), suggesting that workers in their early $60 \mathrm{~s}$, who may be in a transitional period between full-time work and retirement, are particularly eager to seek out new experiences and challenges. With the oldest Baby Boomers turning 60, this desire to "try something new" could portend a significant wave of departures in the next few years, as many older Boomers set out on different careers - yet another reason why employers need to be paying close attention to workplace culture and the retention of retirement-eligible employees. Further comments from the in-depth interview suggest that ICT training is the top wanted challenge for both employers and employees. From the point of employers, ICT skills help to make their business in a more effective way. From the point of employees, ICT skills could be used in building up their social network in the future.

Across all three age groups (50-55)(56-59)(60-65), in fact, employees underscored the importance of flexibility and the opportunity to learn, grow and experience something new. One interesting point is that almost all respon- 
dents in the survey agreed that the use of ICT is very important in order to help them to achieve their goals meeting changing opportunities in their retirement age. It is a kind of catalyst for such transition when they are ready to enter into their next stage. A large number of aged workers will have to adapt their skills, develop a voluntary and pro-active attitude towards lifelong learning and become familiar with new ICT tools if they wish to be further included into the labour market. Among the most frequent reasons chosen by respondents:

- "To enjoy their life with the latest technology"

- "To spend more time with old friends and younger generation with the latest communication methods eg: e-mail, facebook,etc"

- "Wanted to try something different with the latest technology"

- "To become self-employed or start a business"

- "New job opportunity came up"

- "Needed more flexibility to balance work/personal life”

This means that the national institutions in charge of helping and accompanying unemployed aged worked workers in their way back to employment will have to evolve themselves by understanding in concrete what is happening in the labour market and the skills that are required. The implications are clear for employers who want to retain talented knowledge workers nearing retirement age: give older workers what they crave - new experiences and challenges with appropriate training support. Instead of allowing experienced workers to "retire" - and perhaps re-enter the workforce in a new job capacity sometime in the future - employers should consider appropriate ICT training as a possible solution for increasing retention of experienced employees who still want to work.

One of the most unexpected findings from this study was the clear trend toward showing interest in the use of technology supporting to stay active and engaged. Some $35 \%$ of respondents age 55-59 reported that they work enjoy life and connect with friends and younger generation with latest technology. For 60-65 year-olds, a similar figure is also observed.

\section{B. ICT providers interviewed}

Another interesting feature that emerged was the way in which the people interviewed had directed their own professional development and fostering of expertise in respect of the role of ICT Learning in corporate learning delivery.

There were some indications of issues that needed to be addressed.

Barriers mentioned in the interviews included:

The sheer busyness of staffs in meeting a growing range of tasks

This sometimes led to professional development being crowded out by activities seen as more immediate priorities (i.e. not core business), suggesting the need for renewed affirmation of the essential nature of professional development in changing times.

Some provider administrators not being positive about the use of ICT

There are dilemmas for administrators about the use of technology, and especially web 2.0 technologies that are banned in many education institutions. Some administrators were reported to be blockers to the use of ICT, perhaps for cost reasons, and

Small providers being without a capacity to develop a professional support system.

Small private providers seldom had the capacity or resources to develop such systems. Those interviewed mostly were self taught, some with the assistance of software companies and/or informal mentoring assistance from a public provider.

\section{Market fragmentation and lack of interoperability}

The market environment for ICT products and services relevant for older people is highly diverse, and this diversity has led to complex and fragmented demand and supply, lacking the adequate scale for a profitable uptake and a niche approach by companies. Different actors are involved in the development and provision of ICT inclusive products and services, and a variety of social and local factors of technological, psychological, sociological, political, economic nature influence uptake. This creates market barriers in terms of commercialisation and in terms of overcoming technical barriers notably interoperability. Information provided here should include:

- How is procurement of ICT solutions for ageing organised and which actors are consulted in the process? Any international collaboration on this?

- Are there special market studies carried out in the various MS aiming to collect and analyze data on ICT and ageing products and services and focusing on problems i.e. how to reduce prices for users of ICT products and services?

- Are there statistics/estimates of the number of elderly people actually using ICT tools and services for independent living/active ageing technologies existing in the respective MS? If yes, this information should be provided.

\section{New Views on Retirement}

1) Returning to Work - Willing to learn, to contribute and to be active

For many aging Boomers especially those well educated individuals show new interest in and flexibility surrounding employment beyond the age of 60 - are reshaping the very definition of "retirement." With a much better retirement saving plan comparing with the past, people are no longer to work beyond the age of 55 for the financial reason only, but instead many of them comment that "they choose to work as a way to keep mentally and socially engaged." When asked if their concept of "retirement” had changed, over $60 \%$ of older Boomers (age 55-59) said "yes." Another frequently cited observation was that their new concept of retirement is not stop working, but rather work in a more flexible manner and many of them are willing to learn new skills such as ICT to equip themselves to prepare for the future challenge ahead. Over $80 \%$ of the respondents want to tailor their education programs according to life stage to help preparing and planning for their new concept of retirement.

The authors found mature age workers engaged in ICT Learning in our study included:

- Large numbers from the 'learn to earn' market segment seeking upward career moves or a change of ca- 
reer. Others were return to work aspirants or retirees involved in e-learning for income supplementation purposes.

- A few were in the 'learning leaders'group, undertaking ICT Learning for personal reasons, including for volunteering. These were generally seen as willing learners rather than forced learners, although often they had low literacy levels and needed training in ICT skills.

- A majority with regard to e-learning could be said to fall into the learner categories of 'only if you make it easy'or 'almost there' but these usually needed to overcome some barriers.

\section{DISCUSSION}

According to the survey results, until now the majority of employers are not sufficiently aware of the potentials ICTs have with regard to enhancing the productivity of older workers on the one hand and increasing job satisfaction of employees on the other hand. There is still an unexpectedly high resistance to adopt ICTs in the working process and a large number of elderly employers are not adequately aware of the potentials they can have to increase work-life balance of (older) employees. This is also recognised by older people themselves since they feel that employers do not understand and adequately address their particular needs. Furthermore, the survey results reveal that, with regard to ICT, much more basic issues had to be addressed rather than focusing on further exploiting the wide range of contributions ICTs can make to better adapt to age-related requirements, An example from the survey is the computer buddy approach, which has been very successful in developing ICT skills of older employees: Computer buddies are volunteers with reasonable basic skills who teach employees of the same age. The computer buddy approach is a very effective way of peer assisted learning. It helps older employees in getting over the initial confidence and skill barrier and provides flexibility in response to individual needs. Some previous findings on the learning environment preferences of the elderly learners include:

- creating a safe, non-threatening environment with a different approach to learning in order to motivate and engage the diverse range of mature age workers [6].

- enabling factors include peer support, self-paced learning, mentoring, and tutoring [7].

- effective use of virtual learning environments can enable many older students to gain confidence [8].

- the potential of building online communities among older learners who are not oriented to computers [9].

In term of learning content, older employees want it more tailored to their personal need. ICT enables more individualised approaches to learning to be progressed with increase regard to individual need. The above survey results are confirmed with a report undertaken by the British Educational Communications and Technology Agency (Becta) on new technologies valuable in providing insights on the general direction of development of the elearning role [10]. Becta in this 2007 review of new technologies in education had concluded that e-learning had become more personal, social and flexible. The authors believe that these attributes are particularly relevant to the diverse needs of mature age workers (and older learners generally) so that we have concluded that e-learning with the support of ICT tools has very significant potential in meeting the needs of mature age workers, including people returning to the workforce, that should be harnessed in comprehensive strategies to maintain the skill levels, motivation, and employability of mature age workers.

\section{A. Focus on informal learning}

Survey results also find that it seems difficult to keep older worker learning participation at a reasonable level, due to continuous work pressure and their willingness. The same finding is observed in a study conducted by Oberski cum suis in 2000 [11]. Both of employers and workers mentioned before in this pin the survey that, because of time pressure, much of the learning that is present in organisations can be characterised as informal learning, aimed at solving work-related problems. This is not the only reason to argue for the importance of more attention for informal-learning processes in organisations aimed at learning around problems that really matter. Workers not only seem to be prepared to invest more of their time, the learning outcomes seem to take root more. Workers learn when they feel they need to (so-called just-in-time learning). A positive side-effect mentioned by employers is that managers seem to be more inclined to implement ICT when it is aimed at dealing with work-related problems. However, one should avoid that informal learning gets an ad hoc or a non-structural meaning. In order to address this, the informal-learning environment should be aimed at developing certain competencies and skills that are necessary, but the environment should be embedded in the daily practice where urgent work related problems need to be addressed.

\section{B. ICT learning in small and medium enterprises}

One interesting fact for Hong Kong is that many companies here are small and medium enterprises (SMEs). In Hong Kong, SMEs are the backbone of regional prosperity and employment. SMEs are relatively flexible and quickly adapted to change, and in this light, seen as key organisations for driving innovation and to promote employment.

Employees’ professional development is often seen as a precondition to achieve innovations and to compete with other companies as indicated in the survey. However, survey results reports that small enterprises are often not able to develop or finance learning initiatives independently.

Regarding to the interview, the following points for discussion can be made:

- First there seems to be little time for learning available. Everyday work and pressure are often addressed in favour of structural dedicating time for workplace learning. For most SMEs in Hong Kong, it is harder to disengage workers from their daily practices in order to attend a formal training or a course than in large enterprises. Therefore, much of the learning and education in SMEs is spontaneous or unplanned, often focused on dealing with or solving daily work-related problems

- In the design of learning environments there seems to be little consideration of issues such as learner motivation and involvement. This is often linked with the large drop-out number of participants halfway during 
a course or training. These participants feel that the training does not deal with the problems they encounter in their work and the issues they would have liked to address during the training

- Professional development in SMEs still has a low priority, regardless of the increasing strategic value of this sector in the economy. This is not only due to daily-work pressure, but also to the lack of knowledge about training and development by the management or the owner of the company and a related conservative attitude

An evaluation study on our online survey shows that some workers indicated that they were unfamiliar with the Internet technology, that the connections were instable and slow, have unsuitable soft- and hardware to download files, and that there was limited technical support. These problems are not specific to implementing ICT for learning at the workplace in SMEs; also within formal educational settings these complaints are echoed. More specific for SMEs dealing with local business only, especially those were run by a traditional family, it seems that not all workers have a computer and that knowledge, skills and especially the attitude required to use ICT Learning technology are not widely spread. This is particularly true for so-called micro companies.

Our case shows that there is little support from public bodies and branch or umbrella organisations like chambers of commerce for the implementation of ICT Learning in SMEs in Hong Kong. The implementation of ICT Learning depends on knowledge, skills and attitudes of the managers or owners of the companies involved. The main reason for not using or implementing ICT Learning was due to the fact that managers had either insufficient time or financial resources to acquire the necessary skills and to utilize the possibilities of ICT Learning.

The authors would like to suggest that medium-sized organisations with a training and development staff should appoint a (part-time) staff member who is responsible for ICT Learning in the company. This person could then focus on the following tasks:

- Assess workers' needs when it comes to training and development;

- Determine in what way ICT Learning can address those needs;

- Study the content and pedagogical approach of different ICT Learning products;

- Manage the relationships with the companies that offer ICT Learning;

- Manage the budget available for ICT Learning;

- Collaborate with the system administrator within the organisation;

- Acquire necessary skills and knowledge on ICT Learning and share these experiences with colleagues in the company, and

- Stimulate and promote the use of ICT Learning.

\section{Partnerships with companies and institutions}

From the above mentioned experiences with workplace learning in small organisations raises the argument to establish networks or partnerships to overcome some of the barriers discussed. The structure of these companies make them adaptive to change. However, this often requires a change in the way companies collaborate with each other. SMEs are increasingly dependent on relationships between other companies, larger companies, branch organisations and public bodies to be engaged in task that otherwise would be beyond their individual reach. Partnerships between companies (also called consortia) make it possible to employ and pay for ICT Learning initiatives together. Academic institutions not only have the knowledge and experience with using and implementing ICT and its infrastructure, they also want (or need) to establish collaborations with business organisations. Economics provides some arguments as well for the importance of networks or partnerships between companies, organisations and institutions. Essential in this view is that the required need for innovations and economic developments in some regions is not only driven by demands of the market, efforts of multinationals, and government control. The ability to interact and communicate about innovations and investments in professional development influence future economic developments and generate employment.

\section{Makes ICT-learning more accessible}

A final issue in ICT Learning in SMEs in Hong Kong is the accessibility of learning environments and content. This is problematic as one of the reasons for using ICT Learning to make training and development more accessible to a wider group of people. An explanation for this might be the fact that the production of ICT Learning materials is in the hand of private companies. At the moment it is doubtful whether these private bodies are willing to take the risk to unclosed ICT Learning for workers, who in general have little experience with learning at the workplace in combination with ICT. Perhaps academic institutions and governmental bodies can play a role in the production of ICT Learning applications and courses.

\section{CONCLUSION}

\section{A. Impacts and Benefits of ICT for Older Workers}

As noted above, ICT can contribute to making work more age-friendly in a number of ways:

- Quality of work \& employment: Jobs involving ICT tend to be better quality, involving non-repetitive, non-monotonous tasks and low levels of physical strain. Introduction of ICT is often associated with improved job quality, provided there is proper preparation and support. However, there is evidence for some intensification of ICT-based work, making older workers using computers less likely to have a relaxed workspace compared to other older workers. Excessive hours, information overload and increased complexity can lead to stress, while reduced human and social contacts can lead to feelings of isolation. Physical impairments (e.g. repetitive strain injury, musco-skeletal injuries and eye strain) can also increase.

- Engagement in the labour market: There is mixed evidence on whether diffusion of ICT has encouraged early retirement of older workers. So far any direct effects appear relatively small. Lack of ICT skills may discourage some older workers from seeking to return to work. And as noted above, quality of work is strongly linked to decisions to remain working or to stop. Links may increase in the future, as re- 
cruiters place a higher premium on older workers' ICT skills and workers themselves recognise the value of these skills within the labour market. ICT can also play a role in helping to capture the knowledge and expertise of senior workers when they do retire.

- Caring \& other informal work / work-life balance: While we will not consider this issue in detail, it is important to note that the flexibility offered by ICT can improve work-life balance for carers and those involved in other informal/unpaid work. Among other opportunities, it offers them the possibility of teleworking and of using ICT for providing care remotely from their workplace.

\section{B. Good ICT Learning practices: the key to success}

The authors have identified a range of key features which students believe constitute a high quality online learning experience. The teachers and managers interviewed were asked to comment on these success factors. There was general agreement that the critical success factors relate to:

- responsive teachers

- quality materials

- accessibility and convenience

- interaction and communication

- responsiveness to preferred learning styles

- valid assessment

Several interviewees picked up distinctive features of the ICT Learning role with mature-age workers and suggested a few additional success factors as well as expanding on the success factors listed above.

The teachers and managers interviewed were of the view that anyone can develop the necessary skills to undertake ICT Learning. There was a strong consensus across the interviews on a few key points related to effective ICT Learning engagement and delivery strategies and resources for the mature aged. This suggests the following guidelines in provision for older workers:

- view the mature aged as they really are

- use blended learning strategies preferably

- be clear about the reasons for and benefits of the learning; relate it to the previous life experience of older learners

- conduct a careful orientation/induction process

- build confidence, self esteem, and a sense of capacity to succeed (identity capital) in the orientation phase

- provide supporting, engaging, and rewarding learning environments;

- take older learners slowly along the technology use for learning pathway, and give them time to become familiar with new technologies; do not force the pace with web 2.0 technologies such as blogs and wikis but do test their suitability to your particular group of mature aged

- build motivation for lifelong learning and

- look at strategies to link e-learning into workplaces

ICT Learning can be a powerful tool in the development of more flexible strategies in adapting to the needs and preferences of older people. The authors would like to conclud that trends in the application of learning technologies are moving in the direction of strategies that are more personal, social and flexible. These are attributes that are ideally suited to the preferences of mature age learners who bring a wide range of individual needs, much life experience, and a wish to learn at a time, place and manner that suits their lifestyle and values.

The potential exists to extend the ICT Learning role and impact for mature age workers, building on the findings of this study in a wide range of contexts. This is likely to lead to a deeper understanding of how ICT Learning can be a key tool in adapting the changing ageing workforce landscape to the needs and preferences of learners in a range of contexts, in successive phases of life, and especially at key transition points, such as returning to the workforce after an absence or gradually retiring from the workforce and taking up volunteering activities or changing careers to better suit changing life circumstances.

\section{REFERENCES}

[1] Reday-Mulvey G.Working Beyond 60. Key Policies and Practices in Europe. Great Britain: Palgrave Macmillan 2005 (doi:10.1057/9780230504981)

[2] Ilmarinen J. Towards a Longer Work Career. Ageing and Quality of Life in the European Union. Helsinki: Finnish Institute of Occupational Health. Ministry of Social Affairs and Health 2006.

[3] Tuomi K, Huuhtanen P, Nykyri E et al. Promotion of work ability, the quality of work and retirement. Occup Med (Lond) 2001;51:318-324. (doi:10.1093/occmed/51.5.318)

[4] Tuomi K, Ilmarinen J. Past, present and future of work ability. In: Ilmarinen J, Lehtinen S, ed. Past, Present and Future of Work Ability. People and Work, Research Reports 65. Helsinki: Finnish Institute of Occupational Health 2004; 1-25.

[5] Tuomi K, Ilmarinen J , Seitsamo. J. New dimension of work ability. In: Costa G, Goedhard WJA, Ilmarinen J, ed. Assessment and Promotion of Work Ability, Health and Well-Being of Ageing Workers. International Congress Series. No. 1280. The Netherlands: Elsevier 2005; 3-7.

[6] Gelade, S, Catts, R. and Gerber, R. Securing success: good practice in training people aged 45 and over who are disadvantaged in the labour market 2003.

[7] Taylor, T. and Rose, J. Bridging the Divide: Older learners and new technologies- ICT and older learners: strategies and case studies 2004.

[8] Heaperman, S. and Sudweeks, F Achieving self-efficacy in the virtual learning environment. Paper presented at the Australian Association for Research in Education (AARE) conference 2001, Fremantle, WA.

[9] Schuller, T. The Benefits of Learning: The impact of education on health, family life and social capital, Routledge Falmer: London 2004.

[10] Becta (British Educational Communications and Technology Agency). Emerging Technologies for Learning 2007, Volume 2, Becta: Coventry

[11] Oberski, I., Palomar, A., Nova, C., Ruggiero, E., Herrera, F., Korhonen, K., Osborne, M., \& Davies, P. Evaluating online workbased education for managers in SMEs: Some initial observations. Industry \& Higher Education 2000, 14, 200-203.

\section{AUTHORS}

S. Lam (sancheslam@gmail.com) was with New College, Oxford University. He is now with the Social Sciences Division, Oxford University, UK.

W. Chung (winnie0903@hotmail.com) is with the Social Work Department, Tung Wah Eastern Hospital, Hong Kong.

Submitted December 14, 2009. Published as resubmitted by the authors February $2^{\text {nd }}, 2010$. 